\title{
LIPOMA SUBMUCOSO POLIPOIDE DE LA VÁLVULA ILEOCECAL. INFORME DE UN CASO
}

\author{
POLYPOID SUBMUCOSAL LIPOMA OF THE ILEOCECAL VALVE. REPORT OF A CASE
}

LIPOMA SUBMUCOSO POLIPOIDE DA VÁLVULA ILEOCECAL. REPORT DE UM CASO

\author{
NEIRA JAMES' ${ }^{1}$ MACÍAS J. LÚVER², PAREDES DIEGO², CORONEL R. JHON², NEIRA FÁTIMA ${ }^{3}$ \\ ${ }^{1}$ Universidad de Guayaquil, Guayaquil, Ecuador \\ ${ }^{2}$ Hospital Luis Vernaza, Guayaquil, Ecuador \\ ${ }^{3}$ Omni hospital, Guayaquil, Ecuador
}

Introducción: la submucosa del colon es un sitio raro para la formación de lipomas, que son por lo general clínicamente insignificantes. Objetivo: presentar un caso clínico en el que se produjeron cuadros oclusivos parciales intestinales, secundarios a un lipoma submucoso de la válvula ileocecal, así como hacer una revisión de la literatura. Caso clínico: varón de 49 años de edad con cuadro clínico de 48 horas de evolución caracterizado por dolor abdominal generalizado acompañada de deposiciones líquidas en número de 4 en las primeras 24 horas que luego progresa a la constipación. En las imágenes se observó una invaginación intestinal del colon. La Patología identificó un lipoma submucoso como el intussuseptum, que fue resecada para la mejoría clínica del paciente. Conclusión: el Lipoma Submucoso de la Válvula ileocecal es una neoplasia benigna y rara. El diagnóstico se puede realizar con ayuda de una tomografía axial computarizada donde se demuestre tumoración con densidad grasa.

PALABRAS CLAVE: lipoma, colon, Intususcepción.

Abstract

Introduction: The colonic submucosa is a rare site for the formation of lipomas, which are usually clinically insignificant. Objective: to present a clinical case in which partial intestinal occlusive symptoms were produced, secondary to a submucosal lipoma of the ileocecal valve, as well as a review of the literature. Clinical case: a 49-year-old man with a clinical picture of 48 hours of evolution characterized by generalized abdominal pain accompanied by liquid stools in the number of 4 in the first 24 hours, which then progressed to constipation. In the images an intestinal intussusception of the colon was observed. Pathology identified a submucosal lipoma resembling the intussuseptum, which was resected for the clinical improvement of the patient. Conclusion: the submucosal lipoma of the ileocecal valve is a benign and rare neoplasm. The diagnosis can be made with the help of a computerized axial tomography where a tumor with fat density is demonstrated.

KEYWORDS: lipoma, colon, Intussusception.

Resumo

Introdução: a submucosa do cólon é um local raro para a formação de lipomas, que geralmente são clinicamente insignificantes. Objetivo: apresentar um caso clínico em que quadros oclusivos parciais intestinais foram produzidos, secundário a um lipoma submucoso da válvula ileocecal, bem como uma revisão da literatura. Caso clínico: homem de 49 anos com quadro clínico de 48 horas de evolução caracterizado por dor abdominal generalizada acompanhada de fezes líquidas no número de 4 nas primeiras 24 horas, evoluindo para constipação. Nas imagens foi observada uma intussuscepção intestinal do cólon. A patologia identificou um lipoma submucoso, como o intussuseptum, que foi ressecado para a melhora clínica do paciente. Conclusão: 0 lipoma submucoso da válvula ileocecal é uma neoplasia benigna e rara. O diagnóstico pode ser feito com ajuda de uma tomografia axial computadorizada mostrando um tumor com densidade de gordura.

PALAVRAS-CHAVE: lipoma, cólon, intussuscepção. 


\section{INTRODUCCIÓN}

El lipoma submucoso de la válvula ileocecal, es también conocido como hiperplasia lipomatosa de la válvula ileocecal (HLVI), hipertrofia de la válvula ileocecal o síndrome de la válvula ileocecal. ${ }^{1}$

El primer lipoma colónico fue descrito en 1757 por Bauer, ocupa el segundo lugar entre los tumores benignos colónicos, por detrás de los pólipos edematosos, la incidencia varía entre el 0,035 y el 4\% de los tumores colónicos, siendo más frecuente en mujeres entre los 40 y los 50 años de edad. ${ }^{2}$ Los lipomas de colon son más frecuentes en el ciego (30\%), seguido del colon ascendente y transverso, (18 y 17\% respectivamente), siendo el colon izquierdo el lugar de menor localización. ${ }^{3}$ Algunos autores afirman que los tumores de más de $2 \mathrm{~cm}$ de diámetro pueden ser sintomáticos, con dolor, hemorragia, diarrea o estreñimiento ${ }^{4}$ producido por obstrucción intestinal.

Las oclusiones intestinales por intususcepción se asocian en la mayoría de veces a un tumor intestinal benigno (30-50\%), y en un porcentaje algo inferior (10-25\%), el proceso asociado es un tumor maligno; ${ }^{5}$ es raro que los tumores intestinales benignos produzcan sintomatología, y si la producen es a través de sangrado, dolor abdominal y, en ocasiones, crisis suboclusivas: ${ }^{6}$ no es frecuente que produzcan cuadros oclusivos, y si lo hacen, es a través de un mecanismo de intususcepción intestinal. ${ }^{7}$

El tratamiento definitivo del lipoma de la válvula ileocecal es la exéresis quirúrgica, especialmente cuando produce una situación de obstrucción intestinal aguda o una invaginación recurrente, como en el caso que nos ocupa. ${ }^{8}$ El propósito de este estudio es presentar un caso clínico en el que se produjeron cuadros oclusivos parciales intermitentes intestinales, secundarios a un lipoma submucoso de la válvula ileocecal, así como hacer una revisión de la literatura para describir otras presentaciones clínicas, formas diagnósticas y terapéuticas, ya que no existe mucha información

\section{CASO CLÍNICO}

Paciente de 49 años de edad que acude a la consulta externa del hospital Luis Vernaza de la ciudad de Guayaquil el 22 de julio del 2015, con antecedentes de hipertensión arterial controlada; ingresó por presentar cuadro clínico de más o menos 48 horas de evolución caracterizado por dolor abdominal generalizado de intensidad moderada acompañado de deposiciones líquidas en número de cuatro en las primeras 24 horas que luego progresa a la constipación, vómito en número de uno, en cantidad abundante; malestar general, escalofríos y alza térmica no cuantificada. Refiere que hace tres meses presenta astenia, anorexia y pérdida de peso. Al examen físico presenta palidez generalizada, abdomen blando, depresible, doloroso a la palpación profunda en flanco derecho, donde se aprecia masa de consistencia blanda, dolorosa a la palpación, de aproximadamente $5 \times 4 \mathrm{~cm}$, de superficie irregular, móvil. Ruidos hidroaéreos aumentados, Blumber y Mc Burney positivos. En la Ecografía abdominal se aprecia a nivel paraumbilical derecho, imagen de cuatro centímetros transversalmente que se extiende infra-umbilicalmente con aspecto de diana, que sugiere intestino aperistáltico con edema de sus paredes y de la grasa intestinal (Figura 1).

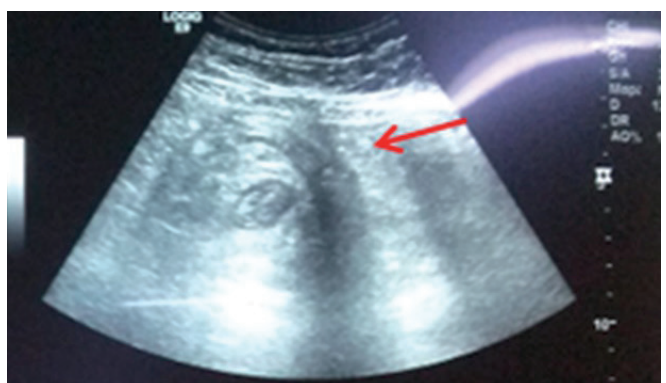

Figura 1. Ecografía abdominal; se observa imagen de $4 \mathrm{~cm}$ transversalmente con aspecto de diana a nivel paraumbilical (flecha).

La TAC Abdominal reporta imagen en diana a nivel de asas intestinales de colon ascendente y de intestino delgado, íleon, que mide $7 \mathrm{~cm}$ en probable intususcepción a nivel de ampolla cecal, paredes intestinales con edema interasas que mide $11 \mathrm{~cm}$, edema de la grasa mesentérica locorregional e incremento de los ganglios mesentéricos locorregionales que miden $1.3 \times 1.3$ $\mathrm{cm}$. (Figura 2).

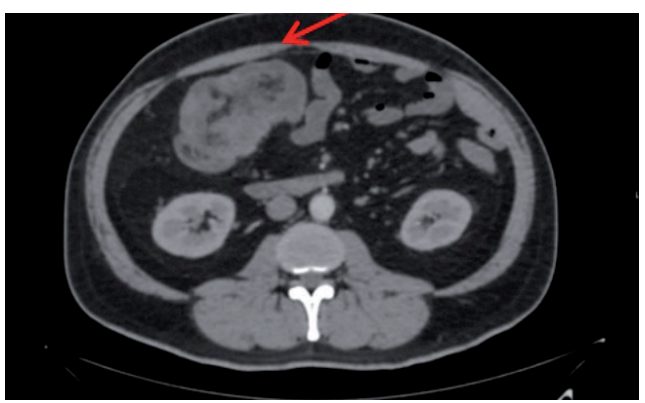

Figura 2. TAC abdominal; corte transversal; se observa imagen en diana a nivel de asas intestinales de colon ascendente y de intestino delgado íleon que mide $7 \mathrm{~cm}$ en probable intususcepción a nivel de ampolla cecal e incremento de los ganglios mesentéricos locorregionales el de mayor tamaño mide $1.3 \times 1.3 \mathrm{~cm}$ (Flecha). 
Se realizó colonoscopía donde se encontró en ciego lesión exofítica de aspecto vegetante, friable de base ancha que incluye válvula ileocecal de 45mm de diámetro, (Figura 3).

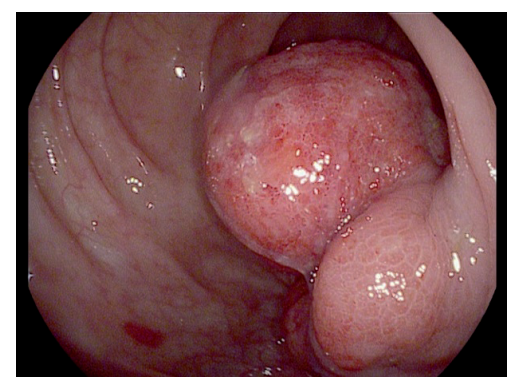

Figura 3. Colonoscopía revela lesión exofítica de aspecto vegetante, base ancha a nivel de ciego que compromete válvula ileocecal de $45 \mathrm{~mm}$ de diámetro.

Durante la laparotomía exploratoria se evidencia masa tumoral de más o menos $7 \mathrm{~cm}$ de diámetro en el ciego que no se exterioriza a cavidad abdominal y ganglios inflamados pericecales, realizándose hemicolectomía derecha + anastomósis ileocolónica con grapadora lineal. El examen macroscópico de la muestra se apreció tumor de aspecto polipoideo de base ancha de $5 \times 4,5 \times 4 \mathrm{~cm}$, multilobulado, rojizo con área central necrótica verdosa, que dista a $12 \mathrm{~cm}$ del borde quirúrgico proximal y $16 \mathrm{~cm}$ del borde quirúrgico. El examen microscópico tumor submucoso constituido por tejido adiposo maduro, (Figura 4). La mucosa suprayacente parcialmente ulcerada, con exudado fibrinoleucocitario y desarrollo de tejido granulatorio.

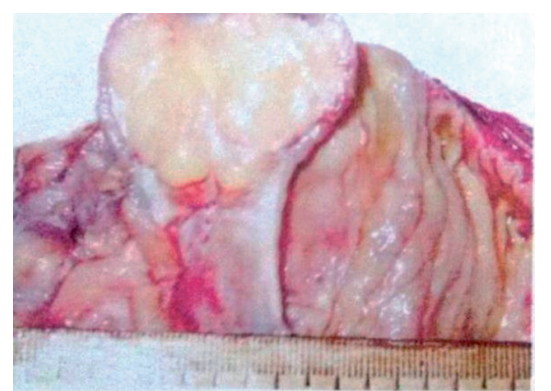

Figura 4. El examen macroscópico de la muestra se apreció tumor de aspecto polipoideo de base ancha de $5 \times 4,5 \times 4 \mathrm{~cm}$, multilobulado, rojizo con área central necrótica verdosa, que dista a $12 \mathrm{~cm}$ del borde quirúrgico proximal y $16 \mathrm{~cm}$ del borde quirúrgico.

\section{I.D.: LIPOMA SUBMUCOSO POLIPOIDE DE LA VALVULA} ILEO-CECAL

Luego de 10 días del postoperatorio el paciente presentó evolución satisfactoria, mejorando su cuadro clínico, siendo dado de alta sin complicaciones relevantes y tratamiento clínico de mantenimiento

\section{DISCUSIÓN}

Los lipomas están dentro del grupo de las neoplasias benignas no epiteliales de baja frecuencia y es la segunda neoplasia más frecuente de colon; son tumores bien diferenciados originándose de tejido conectivo intestinal, con incidencia del $0.035 \%$ a $4.4 \%$, presentándose entre los 50 y 70 años de edad; predomina en raza blanca.

Los estudios de autopsia han demostrado la incidencia de 4 y $5 \%$ en los lipomas colónicos. Por lo general, se producen entre la quinta y sexta décadas de la vida y son a menudo hallazgos incidentales durante la colonoscopía o la cirugía. ${ }^{9}$

Los tumores no epiteliales intestinales son raros tumores benignos que pueden imitar las lesiones malignas. ${ }^{10}$ Menos del $30 \%$ de los lipomas se observan en la literatura que son sintomáticos. ${ }^{11}$ Los Lipomas mayor a $2 \mathrm{~cm}$ de diámetro es probable que sea sintomático. ${ }^{12}$ Los síntomas de lipoma submucoso no son específicos, por lo que el diagnóstico correcto puede ser difícil de alcanzar debido a poca información de estos tumores y la falta detallada de síntomas y signos a menudo representa un desafío para el diagnóstico. Pueden causar sangrado, la obstrucción y la invaginación intestinal.

En el paciente todo lo mencionado previamente es aplicable, evidenciándose que fue un hallazgo que se realizó en el mismo acto quirúrgico, debido a la muy baja frecuencia de tumoraciones de tipo lipomatosa, benigna, que debutan con una obstrucción intestinal y deterioro clínico del paciente. Endoscópicamente tienen la característica apariencia polipoidal amarillo suave, tres signos contribuye al diagnóstico en la colonoscopía que es el signo del cojín (las lesiones submucosas se comprimen fácilmente durante la biopsia, pero rápidamente regresan a su tamaño anterior), el efecto tienda de campaña (al agarrar la mucosa suprayacente con pinzas da una apariencia de tienda de campaña) y el signo de grasa desnuda (grasa que se extruye al realizar la biopsia). ${ }^{13}$

En el enema baritado el lipoma aparece como un defecto de llenado delimitado ovoide. El signo característico es la señal del apretón (el tumor puede deformarse por la presión externa o el peristaltismo). ${ }^{14}$ 
La TAC se considera que es el método diagnóstico definitivo en el reconocimiento de los lipomas de colon; un lipoma intususceptado puede mostrar la apariencia atípica y puede ser confundido con otro tumor. ${ }^{15} \mathrm{El}$ tratamiento de los lipomas de colon puede realizarse con seguridad si endoscópicamente es pequeño. Sin embargo, lipomas de más de dos centímetros, se cree que tienen un mayor riesgo de perforación del colon. Existen criterios de elección para establecer la cirugía por laparotomía o laparoscopía: a) Lipoma con diámetro mayor a $4 \mathrm{~cm}$, b) Diagnóstico preoperatorio incierto, c) Lipoma con aparición de invaginación intestinal, d) Compromiso de la capa muscular.

En el presente paciente se optó por realizar una laparotomía exploratoria con enfoque directo; se resecó la totalidad de la masa tumoral + vaciamiento ganglionar pericólico, ante la fuerte sospecha de malignidad; por ello se realizó una hemicolectomía derecha, evitando riesgos y por seguridad del paciente. La resección es una forma o tratamiento aceptado. Hay menos de una docena de informes de resección laparoscópica de estas lesiones. A partir de estos informes, el abordaje laparoscópico parece ser una opción segura y razonable para la terapia. ${ }^{9}$ Por ello la importancia de transmitir el aprendizaje evidenciado con este caso, siempre teniendo en cuenta la evolución y mejoría de los pacientes, es incuestionable.

\section{CONCLUSIONES}

El lipoma submucoso de la válvula ileocecal es una neoplasia benigna, rara, que puede ser confundida con otras patologías de presentación más frecuente; su incidencia es mayor entre la sexta y séptima década de vida. Puede llevar a cuadros de abdomen agudo que ameritan manejo de urgencia.

El diagnóstico se puede realizar con ayuda de una tomografía axial computarizada donde se demuestre tumoración con densidad grasa. Solo el estudio histológico permite el diagnóstico definitivo. Los lipomas deben ser resecados sólo si producen síntomas, en especial los mayores de dos $\mathrm{cm}$.

\section{DECLARACIÒN DE ORIGINALIDAD}

Declaramos que el contenido del artículo es original y que no ha sido publicado como artículo previamente ni está enviado para ninguna consideración a cualquier otra revista, en su totalidad o en alguna de sus partes.

\section{CONFLICTOS DE INTERESES}

Los autores declaramos que no hay conflictos de intereses.

\section{REFERENCIAS BIBLIOGRÁFICAS}

1. Pastor CV, Valentí I, Poveda P, Martí G, Zozaya J, Baixauli J, Hernández-Lizoain L. Lipomas submucosos de colon. Rev. Esp. Enferm. Dig. 2007;99:299-00.

2. Pareja E, García-Granero M, Zaragoza E, Ripoll F, Checa F. Lipomas submucosos de colon. Rev Esp Enferm Dig 2002;94:367-73.

3. Taylor BA, Wolff. Colonic lipomas: report of two unusual cases and review of the Mayo Clinic Experience, 1976-1985. Dis Colon Rectum 1987;30:888-93.

4. Caterino S, Tiziano G, Mercantini P, Ziparo V. Rectorrhagia caused by lipoma of the hepatic flexure of the colon. A case report and review of the literature. G Chir 2002;23:205-8

5. Holder W Jr: Intestinal Obstruction. Gastroenterol Clin North Am; 17(2): 317-340 (1988).

6. Brophy C, Cahow CE: Primary small bowell malignant tumors. Inrecognized until emergent laparotomy. Am Surg; 55(7): 408-412 (1989).

7. Brzezinski et al: Small-bowel lipoma: an uncommon cause of obstruction. C J S; 33(5): 423-424 (1990).

8. Siddiqui MS, Fatima L, Khatri AR, Muzaffar S, Quraishy MS. Colonic lipoma. J Coll Physicians Surg Pak 2003;13:291-2.

9. Lornie J. Phillips II, Jonathan D. Svahn. Colonic Lipoma, a rare cause of adult Intussusception, World Journal of colorectal Surgery, Vol 3, Article 19: 12-20; 2013.

10. Coyne PE, Teemul T, Dent B, Henderson D, Crabbe R, Garud T. Submucosal lipoma of the large intestine masquerading as a colonic malignancy. J Surg Case Rep, 17-35; volume 2011.

11. Dhar R, Sahu S and Roychoudhury AK. An Interesting intestinal lipoma case. Int J Sci Res; 28-45; March 2014

12. Mnif L, Amouri A, Masmoudi MA et al. Giant Lipoma of transverse colon: a case report and review of literature. Tunis Med 2009; 87 (6): 398 - 402.

13. Philips II LJ, Svahn JD. Colonic lipoma, a rare cause of adult intussusception. World J Colorectal Surg; 43-45; 2013.

14. Zhang H, Cong JC, Chen CS, Qiao L, Liu EQ. Submucous colon lipoma: A case report and review of the literature. World J Gastroenterol 2005; 11:3167-3169.

15. Janevska V, Spasevska L, Dukova B, Janevski V. Intestinal Submucosal Lipomas. Macedonian Journal of Medical Sciences. March 2012; 5:49-54 